\title{
El nuevo rostro de la migración centroamericana en México
}

\section{The new face of Central American migration in Mexico}

\author{
Laura Myriam Franco Sánchez \\ Universidad Autónoma del Estado de Hidalgo, México
}

\section{Resumen}

El objetivo del presente trabajo fue conocer la migración de centroamericanos que se internan en México con la intención de llegar a los Estados Unidos en una migración de tránsito, sin embargo, no todos logran su cometido y se quedan en territorio mexicano. Se trata de un estudio descriptivo donde el interés es conocer sus características y resaltar las peculiaridades de estos grupos de migrantes. Los resultados de esta investigación muestran que la migración centroamericana se ha incrementado en los últimos años; con una tendencia a una residencia más permanente, ubicándose principalmente en el sur del país. Asimismo, demográficamente es una población joven, con bajos niveles de escolaridad y con trabajos de la economía informal.

Palabras clave: Migración, tránsito, Centroamérica, residencia.

\begin{abstract}
The objective of this study was to know the migration of Central Americans who enter Mexico with the intention of reaching the United States in a transit migration, however, not all of them achieve their mission and remain in Mexican territory. It is a descriptive study where the interest is to know their characteristics and highlight the peculiarities of these groups of migrants. The results of this research show that Central American migration has increased in recent years; with a tendency to a more permanent residence, located mainly in the south of the country. Likewise, demographically it is a young population, with low levels of education and with jobs in the informal economy.
\end{abstract}

Key words: Migration, transit, Central America, residence.

Artículo recibido el 12 de noviembre de 2020 y aprobado el 05 de enero de 2021. 


\section{INTRODUCCIón}

$\mathrm{E}$

l estudio de la migración centroamericana no solo centra en el interés académico, sino también se ubica en uno de los temas de análisis geopolítico de mayor debate donde se discuten sus causas y sus posibles consecuencias para llegar a un mejor entendimiento de este fenómeno, en el cual se integran los países de origen, tránsito y destino quienes juegan un papel muy importante dentro de las agendas sociales y políticas de los países.

La migración centroamericana ha sido considerada un hecho relacionado a las disparidades regionales reflejadas en los bajos salarios de los lugares de origen; aunque también es de importancia resaltar las migraciones forzadas preexistentes ocasionadas por cuestiones sociopolíticas, desastres naturales, la inseguridad, entre otras, las cuales han incentivado la migración, la cual ha tenido un fuerte impacto no solo en los países de origen sino también en los países destino; muchas familias se han desplazado en busca de mejores oportunidades de vida (Expansión Política, 2019:1).

El tránsito de migrantes centroamericanos a su paso por México se ha incrementado en los últimos años, y se ha convertido en un tema de suma relevancia, no sólo por los volúmenes de población que implica, sino también por la vulnerabilidad y riesgos que los acompaña en donde se involucra el asedio de grupos criminales y el ser poco cobijados por las instituciones.

Por tanto, este documento busca aportar un análisis y una caracterización de los migrantes centroamericanos residentes en México, donde se plantea la hipótesis de que este grupo de población es considerado de bajo nivel educativo, asentado principalmente en la parte sur de México e involucrado prioritariamente en actividades agrícolas y de baja calificación. Este grupo de población posiblemente salió de sus lugares de origen con el propósito de buscar mejores oportunidades de vida en los Estados Unidos, pero por diversas circunstancias se quedaron en territorio mexicano. Para ello, el trabajo se estructura en tres apartados: el primero presenta los aspectos teóricos de la migración y movilidad, el segundo una breve trayectoria de la migración centroamericana y el tercero analiza sus características. 


\section{CONSIDERACIONES METODOLÓGICAS}

El objetivo de este artículo es analizar las principales características sociodemográficas de los inmigrantes centroamericanos tomando en cuenta no sólo las configuraciones de los mercados de trabajo, sino también algunos elementos sociodemográficos presentes en los entornos en los que esta migración se desarrolla. Es decir, se considera que la migración centroamericana no responde únicamente a los factores económicos o laborales, sino también a los sociales y los demográficos. Por lo anterior, este documento tiene como objetivo reflexionar sobre la importancia de la población centroamericana en México mediante la utilización de técnicas de investigación cuantitativas y cualitativas. Para ello, se ha efectuado una exploración de diversas fuentes de datos para comprender el fenómeno, en un primer momento conocer de manera general la problemática a través del análisis de la información estadística obtenida por la Comisión Mexicana de Ayuda a Refugiados (COMAR), de la cual se pudo identificar el comportamiento que han mantenido las solicitudes de asilo y la importancia de la población centroamericana en estas, así mismo se analizó información del Boletín Mensual de Estadísticas Migratorias 2020, realizado por la Unidad de Política Migratoria, Registro e Identidad de Personas, con la intención de conocer cómo se han otorgado las Tarjetas de Residente Temporal (TRT) y Tarjetas de Residente Permanente (TRP), emitidas y renovadas, según país de nacionalidad. En un segundo momento se realiza un análisis exhaustivo de los microdatos de la Encuesta Intercensal 2015, atendiendo a las preguntas que indagan los procesos migratorios relacionándolas con las variables de sexo, edad, escolaridad, situación conyugal, etcétera. Esta fuente de información es la base de este análisis por su carácter de confiabilidad y los tópicos que aborda, los cuales constituyen una fuente valiosa para estudiar las características de estas poblaciones. Para el caso que nos ocupa, nos permite analizar adecuadamente a los centroamericanos con las preguntas del lugar de nacimiento y residencia relacionadas con diversas variables sociodemográficas.

Para el análisis cualitativo se aplicó la técnica bola de nieve y el criterio de saturación, por lo que se hicieron siete entrevistas a migrantes centroamericanos que llegaron a México en los últimos cinco años, lo anterior en virtud de conocer a fondo el porqué de su migración, así 
como las causas que la originaron; además de saber las características laborales de esta población residente en México.

\section{MigRaCión}

La migración es uno de los agentes de movilidad más importantes entre sociedades y naciones. Ha sido una constante a lo largo de la historia y tiene repercusiones para los migrantes, los lugares que dejan y los que los acogen (Romero \& Lugo, 2018: 7). De ahí entonces que, la movilidad es una de las prerrogativas fundamentales del ser humano desde tiempos remotos, que ha ido cambiado a lo largo de la historia, por lo cual se puede afirmar que está históricamente determinada (Ramírez, 2015), así mismo, es en sí un tema complejo por entender y debatido por sus concepciones, las cuales han tenido diferentes manifestaciones en los territorios y por ende en la calidad de vida de sus habitantes. Este concepto de movilidad está estrechamente relacionado con los desplazamientos de las personas y las repercusiones de estos movimientos en sus territorios, por ello la migración es comprendida como una de las expresiones más claras de éstas, en donde tanto sociedades como naciones se ven afectadas en su propia vida, así como en los lugares de destino de estos migrantes (Bautista \& Ibarra, 2014).

La movilidad en los territorios se vuelve cada día más compleja lo que ha conllevado al incremento de los flujos de personas este fenómeno no solo se explica bajo el concepto tradicional de la migración, por ello es conveniente introducir el de movilidad humana, el cual refiere a personas emigrantes, inmigrantes, solicitantes de refugio y asilo, refugiadas y asiladas, apátridas, migrantes y desplazadas internas, víctimas de trata y tráfico de seres humanos y sus familias (Carrasco, 2013).

La movilidad se convierte en parte de la vida del ser humano, se expresa en el impacto en los ámbitos políticos, económicos y culturales, así como en el modus vivendi de los habitantes originarios. Las relaciones funcionales entre los diferentes territorios, hacen inminente el flujo e intercambio de bienes, servicios, y población, la cual al relocalizarse las actividades económicas y cambiar la dinámica de las ciudades enfrenta procesos como cambios de residencia, falta de infraestructura o altos costos del suelo que encarece el acceso a vivienda en los lugares de origen, así la falta acceso a ciertas condiciones de vida hacen que la población busque alternativas asumiendo los costos de un cambio de residencia, ya que al moverse a otras ciudades de mayor 
tamaño y con infraestructura creciente aseguran educación, servicios de salud, servicios públicos de calidad, mayor seguridad; pero a su vez asumen en gran medida el hecho de buscar nuevamente un empleo o bien de trasladarse al trabajo que ya tenían, es así como la mayoría de desplazamientos actuales se realizan en busca de un empleo asalariado.

Se identifica tres características de este sistema migratorio, en las cuales enfatiza la existencia de una alta movilidad: 1) alta movilidad poblacional - privilegiadamente unidireccional de sur a norte, tanto de mexicanos como de centroamericanos que desean estar en Estados Unidos-; 2) posicionamientos y regulaciones migratorias que afectan a la región en su conjunto, pero que provienen esencialmente de Estados Unidos; y 3) la situación económica, social y cultural de cada país involucrado define la posición, acciones y reacciones particulares acerca de la movilidad y migración (Nájera, 2015: 255).

En la actualidad la movilidad de las personas tanto fuera como dentro de un país ha permitido conocer diversas esferas de análisis del fenómeno de la migración, que no solo se ubican como el cambio de residencia. Por ello, los movimientos poblacionales en el mundo no solo implican considerar como tal a la migración sino también a aquellos desplazamientos de corta o larga duración que no implican un cambio de residencia. Por tanto, la migración y la movilidad no puede ser entendida una sin la otra, debido a su complejidad, el contexto en el que se desarrollan y las consecuencias de estos movimientos, los cuales se transforman, adquiriendo nuevas características que implican analizarlos desde diferentes perspectivas y horizontes, por lo que es de vital importancia la implementación de diversos instrumentos que permitan medir este fenómeno, sus continuidades y transformaciones (Consejo Nacional de Población, 2016).

Por su parte el concepto de migración de tránsito empezó a utilizarse a partir de la década del noventa, cuando el concepto de país de tránsito emergió en el lenguaje migratorio como un elemento intermedio entre país de origen y de destino (Álvarez, 2011). México se ha ubicado como un territorio estratégico de tránsito a los Estados Unidos por parte dela población centroamericana; sus recorridos pueden durar semanas, meses e inclusive años, aunque algunos jamás llegan a su destino. Sin embargo, pese las medidas tomadas por Estados Unidos y México para impedir esta migración, y con la administración de 
Trump, y las políticas restrictivas implementadas, persiste una alta movilidad de cruces de poblaciones centroamericanas que, si bien, tienen como objetivo llegar a Estados Unidos, algunos de ellos se asentaran a lo largo del territorio mexicano.

\section{TRAYECTORIA DE LA MIGRACIÓN CENTROAMERICANA}

\section{Caravanas de migrantes}

Centroamérica ha tenido períodos en su historia caracterizados por dictaduras y la presencia militar, aunado a problemas de pobreza y desigualdad en sus sociedades. Alrededor de los años de 1970 y 1980 la migración respondió principalmente a la inestabilidad política, consecuencia de la represión y los conflictos armados. En los años de 1990 fue originada principalmente por cuestiones económicas. Actualmente la migración responde a las dinámicas transnacionales, donde grandes masas de población provenientes de Centroamérica se han trasladado en la búsqueda de llegar a los Estados Unidos (sin poseer algún estatus legal), impulsados por la violencia, la reunificación familiar y la búsqueda de mejores oportunidades laborales.

Las caravanas de migrantes no solo es una estrategia de movilidad de estos grupos de migrantes, es una continuidad de procesos históricos de migración, que implican formas de adaptación y de sobrevivir de manera desigual y excluyente. Surgen debido a la afluencia migratoria siendo esta una manera de expresar este fenómeno de una manera más acentuada; estos movimientos se asocian principalmente a cambios estructurales relacionados con la demanda por mano de obra poco calificada, la inseguridad y violencia en sus países de origen, así como también las cuestiones políticas de las cuales son víctimas. La característica principal es que se realiza a pie caminando del lugar origen al lugar destino además de ser masiva. Estos desplazamientos no son nuevos pues se han efectuado a través del tiempo desde Centroamérica hacia los Estados Unidos, sin embargo, es a partir de octubre del año 2018, cuando se intensifican de manera importante.

Las redes sociales han sido un punto de lanza para incentivar estos movimientos ya que es a través de ellas que se realizan las convocatorias a las personas a migrar a los Estados Unidos teniendo como lugar de tránsito a México. El viajar en grupo consideran es una ventaja ya que les proporciona mayor protección, siendo menos vulnerables a delitos y abusos que pueden enfrentarse en su camino, además de 
tener asistencia gubernamental y de otras instituciones, y menos costos de viaje, evitando ser víctimas de coyotes o traficantes, que engañan a los migrantes para cruzarlos por otros países. Estas caravanas de migrantes se conforman por un grupo de población donde viajan a lo largo del territorio hombres, mujeres, adultos mayores y niños en menor proporción, conformando un grupo diverso, y en algunos casos también se unen a este viaje familias completas, todos ellos desconociendo los riesgos a los cuales pueden enfrentarse a lo largo de la ruta y cuyo único fin es una idealización de la migración, persiguiendo el sueño americano.

La Organización Internacional para las migraciones (OIM) señala cuatro ejes para atender a los miembros de la caravana:

1. Entrega de información. Se informa de los servicios que pueden acceder a lo largo de la ruta; estos pueden ser de albergues, asistencia medica, etcétera.

2. Fortalecimiento a las capacidades de las autoridades que estan atendiendo a las personas migrantes de las caravanas. Para ello, la OIM ha otorgado kits de higiene y alimentación.

3. Facilitar el retorno voluntario asistido a aquellos migrantes que deciden regresar a sus lugares de origen. La OIM facilita un regreso seguro y digno, para aquellos que de manera voluntaria decidan regresar ya sea por condiciones de salud, dificultades en la ruta, o demasiada espera de documentación.

4. Recolecta de información sobre la población. Este eje considera la obtención de un perfil del migrante, de manera que pueda asistirle eficazmente (Organización Internacional para las Migraciones (OIM), 2018).

El tránsito de migrantes centroamericanos ha adquirido gran importancia en los años de 2018 y 2019, el flujo de inmigrantes y solicitantes de asilo en la frontera de México y Estados Unidos se incrementó, colocando al límite al sistema migratorio estadounidense. Sin embargo, esta historia de la migración no es nueva pues deviene de varias décadas atrás.

Centroamérica ha sufrido conflictos armados de manera directa e indirecta, en la década de los años ochenta emergieron diversas guerras civiles, así mismo estas causaron medio millón de muertos y millones de personas que tuvieron que desplazarse de forma interna o a otros países (López, 2018). El Salvador, Guatemala y Nicaragua vivie- 
ron conflictos bélicos que generaron un ambiente de crisis política y social en esos lugares, y que propiciaron un incremento importante de migrantes centroamericanos a Estados Unidos. Es en esta época en donde se realizaron múltiples desplazamientos, además de que se vivió una inestabilidad económica e inseguridad para las poblaciones, mismas que, aunque estos conflictos fueron cesando formalmente tras la firma de tratados de paz, aún persistieron de manera informal, dando pauta a la migración ilegal hacia el norte, de ahí que para los años de 1980 y 1990 esta población se triplico en territorio estadounidense.

Además, derivado de los desastres naturales vividos en Honduras, El Salvador y Nicaragua se designó la TPS (Estatus de Protección Temporal), que sería una condición migratoria especial otorgada por el gobierno estadounidense a la población de aquellos países que tengan alguna condición especial de carácter armado, de desastres naturales u otra condición, lo anterior les daría una protección temporal a ser deportados. Sin embargo, en el año 2017 y principios de 2018, Donald Trump señaló la no renovación de las TPS para estos tres países (Migration Policy Institute, 2019).

Las recientes migraciones de centroamericanos de los países del triángulo norte Honduras, Guatemala y El Salvador emergen de una migración forzada, como consecuencia de las condiciones de precariedad, falta de empleo, y la carencia de buenos salarios que persisten en estos países, la violencia que se vive en sus países e inclusive algún desastre natural del que hayan sido participes. Los tres países, tienen una población conjunta de alrededor de 32 millones de habitantes, poseen una estructura económica, política y social precaria, una realidad fuertemente vinculada a los estragos causados por los largos conflictos armados en la región entre 1960 y 1990.

De acuerdo con información proporcionada por la Oficina de Aduanas y Protección Fronteriza de los Estados Unidos en el año 2018, han aprehendido a un alto número de niños no acompañados, alrededor de 38,000 y casi 104,000 personas que formaban parte de una familia de origen salvadoreño, guatemalteco y hondureño quienes se desplazaron a Estados Unidos cruzando por México. Además, aproximadamente 58 por ciento de niños no acompañados y 49 por ciento de individuos eran de origen guatemalteco. Ya para el año 2019 estos arrestos se incrementaron a 363,000 , cifra que se triplico para este año, cabe señalar que estas poblaciones han solicitado asilo. 
México se ha convertido en un afluente importante del tránsito de personas a los Estados Unidos, debido a que posee una ubicación estratégica con el vecino país. La globalización ha permitido la movilización de personas en todos los territorios y México no es la excepción ya que en él han llegado migrantes de diversas nacionalidades. Sin embrago, los centroamericanos es el grupo más representativo de todos ellos. En el año 2019, el Instituto Nacional de Migración (INM) en el país aseguró un total de 186,750 extranjeros irregulares, de los cuales 123,797 eran de Centroamérica, es decir, 82.7 por ciento del total; las principales entidades en donde se han ubicado estos extranjeros fueron Chiapas, Veracruz y Tabasco (SEGOB, 2019). De acuerdo con información del Instituto Nacional de Migración la cifra de migrantes se incrementó en 131.9 por ciento. Además, se resaltó la migración de niños de 0 a 17 como puede verse a continuación: Honduras, 17,034; Guatemala, 10,069; El Salvador, 3,745 y de otras nacionalidades, 2,274. Además, que se presentaron ante la autoridad migratoria 33,122 niños, niñas y adolescentes, y de éstos, 24,597 , eran acompañados de familiares; dejando ver que la cifra de niños solos fue de 8,525, provenientes principalmente de Honduras(Instituto Nacional de Migración, 2019). Lo anterior también tuvo efecto en Estados Unidos donde centroamericanos documentados o irregulares se incrementaron de manera masiva de acuerdo a datos de Migration Policy Institute, donde señala que en 2015 había 3.5 millones de centroamericanos inmigrantes principalmente provenientes de El Salvador, Guatemala y Honduras, representando alrededor de un 8 por ciento de los 43.3 millones de inmigrantes en Estados Unidos (Colegio de la Frontera Norte, 2019).

\section{Estatus legal de la población centroamericana}

El aumento de la migración sin duda alguna tiene múltiples causales, sin embargo, las desigualdades y los desequilibrios económicos, políticos y sociales son los más predominantes; el inequitativo reparto de la riqueza y la desigual atribución de derechos existiendo una brecha muy amplia entre los países en vías de desarrollo y desarrollados, aunado a las crisis económicas y los cambios en las políticas las cuales han originado que incremente la movilidad de la población entre países, quienes han dejado atrás sus lugares de origen en busca de un lugar destino donde puedan acceder a mejores oportunidades de vida, muchos migrantes centroamericanos buscan como fin último llegar a Estados Unidos, aunque a su paso por México muchos deciden que- 
darse a radicar ya sea porque la incertidumbre, los peligros, las leyes o cualquier obstáculo les impida continuar su camino, de ahí que soliciten refugio y asilo o busquen un estatus legal para vivir en el país.

En la Declaración Universal de Derechos Humanos se consagra el derecho de todas las personas a buscar asilo y disfrutar de él. Los refugiados son personas que han huido de su país debido a fundados temores de ser perseguidas por motivos de raza, religión, nacionalidad, opiniones políticas o pertenencia a determinado grupo social. Esto incluye la persecución tanto por parte del Estado como por parte de agentes no estatales (por ejemplo, familiares, vecinos, grupos armados, vigilantes parapoliciales, bandas de delincuentes) cuando el Estado no puede o no quiere brindar protección frente a ese perjuicio (Naciones Unidas, 2020: 1).

El aumento de migrantes durante los años 2018 y 2019 implicó que las solicitudes de asilo y refugio pusieran a prueba la capacidad del sistema migratorio tanto en México como en Estados Unidos. Aunque debe señalarse que esta migración data de una larga tradición histórica. Sin embargo, en su camino muchos migrantes se quedan en México, ya sea como refugiados, residentes temporales o permanentes.

El refugio y el asilo fundamentados en la Ley sobre refugiados, protección complementaria y asilo político instaura los requisitos y mecanismos a seguir para el otorgamiento de éstos, así como también señala la atención a personas que solicitan y adquieren estas condiciones; esta legislación ha facilitado los procesos de asilo y refugio en el país y consecuentemente al aumento de migrantes centroamericanos que lo han solicitado (ver Figura 1).

Hoy en día debido a los problemas de inseguridad y violencia muchas personas centroamericanas han ido en la búsqueda de mejores oportunidades mediante solicitud de asilo o refugio, con residencia temporal o permanente o simplemente de manera ilegal dentro del país.

Me golpeaban la puerta, me insultaban, gritaban que debía pagar el impuesto de guerra que yo debía saberlo, me decían que abriera la puerta, ese día tome la decisión tome una mochila y eche lo que pude y salí corriendo era la madrugada, iba sola con mi hija de dos años, tome el primer autobús, cruce la frontera y llegue a un albergue en Chiapas, yo no tengo el sueño americano yo estoy bien aquí (Entrevista a Teresa Bautista, con la autora, 1 de abril de 2020).

La mara me dijo la única manera que vas a salir tú y tu familia será en un ataúd, no había manera o nos íbamos o moríamos, solo queríamos huir; 
salimos mi esposa y mis dos hijos sin avisar a nadie ni llevar a nada, estoy arreglando mis papeles pues no quiero me deporten, me voy a quedar en Tuxtla pues aquí como sea estamos (Entrevista a José López, con la autora, 6 de marzo de 2020).

.... ya no podíamos aguantar nos exigían debíamos de pagar y pues nos decidimos a escapar, llegamos a Guatemala y después nos venimos para México aquí en Tuxtla ya solicitamos asilo (Entrevista a Ana Dimas, con la autora, 16 de enero de 2020).

Figura 1. Solicitantes de asilo en México 2013-2019

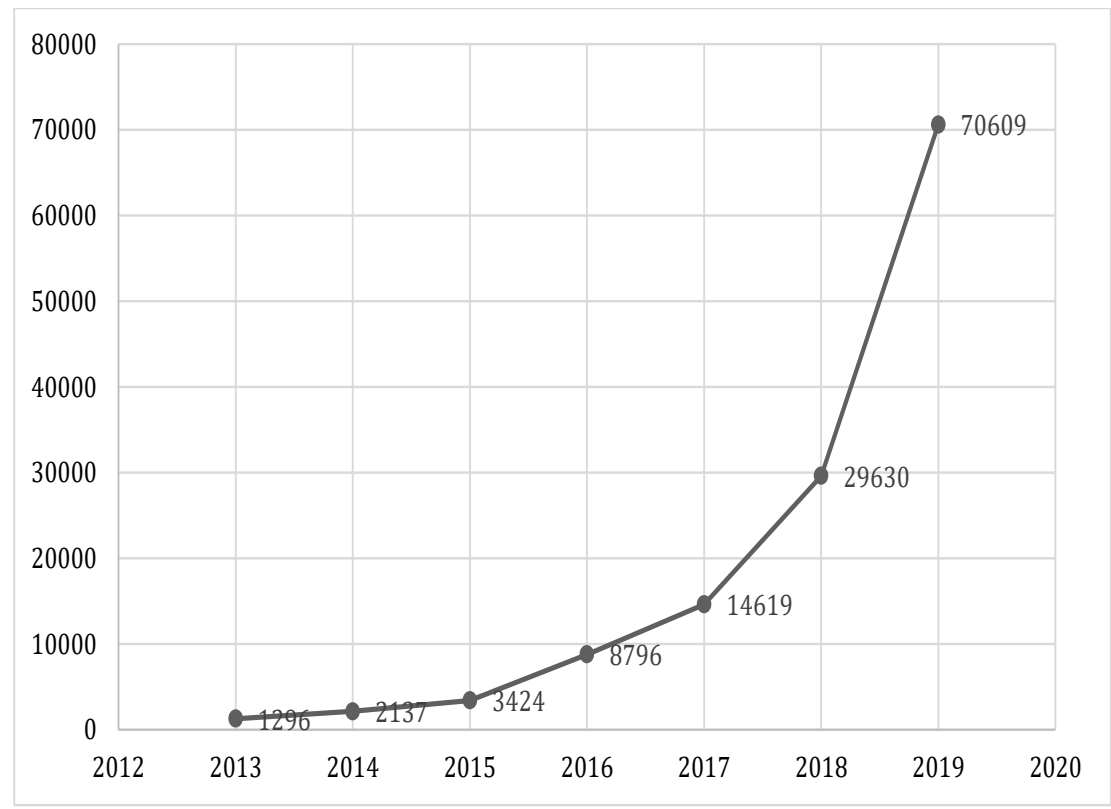

Fuente: Elaboración propia con datos de la Comisión Mexicana de Ayuda a Refugiados, 2019

De acuerdo con las entrevistas con migrantes de tránsito se confirmó que la intención de estos era radicar en México, por lo que les interesaba estar bien en su regulación migratoria. Lo anterior coincide con el aumento de solicitudes de asilo en el año 2019siendo de 70,609 poco más del doble de las recibidas en el año 2018 de 29,630. Además, se destaca que en el año 2019 Honduras (país cuyo proceso migratorio es más reciente en relación a sus flujos) ocupa el primer lugar con 30,187 solicitudes que representan 43 por ciento, es decir, casi la mitad de éstas, seguido de El Salvador con 9,039 correspondiente a 
13 por ciento (país con una historia marcada por violencia, poco crecimiento económico y emigración) y Guatemala con cinco por ciento (país con la más larga tradición migratoria internacional), de ahí se rescata la importancia de estos grupos de población centroamericana (ver Figura 2).

Figura 2. Solicitantes de asilo en México según nacionalidad 2019

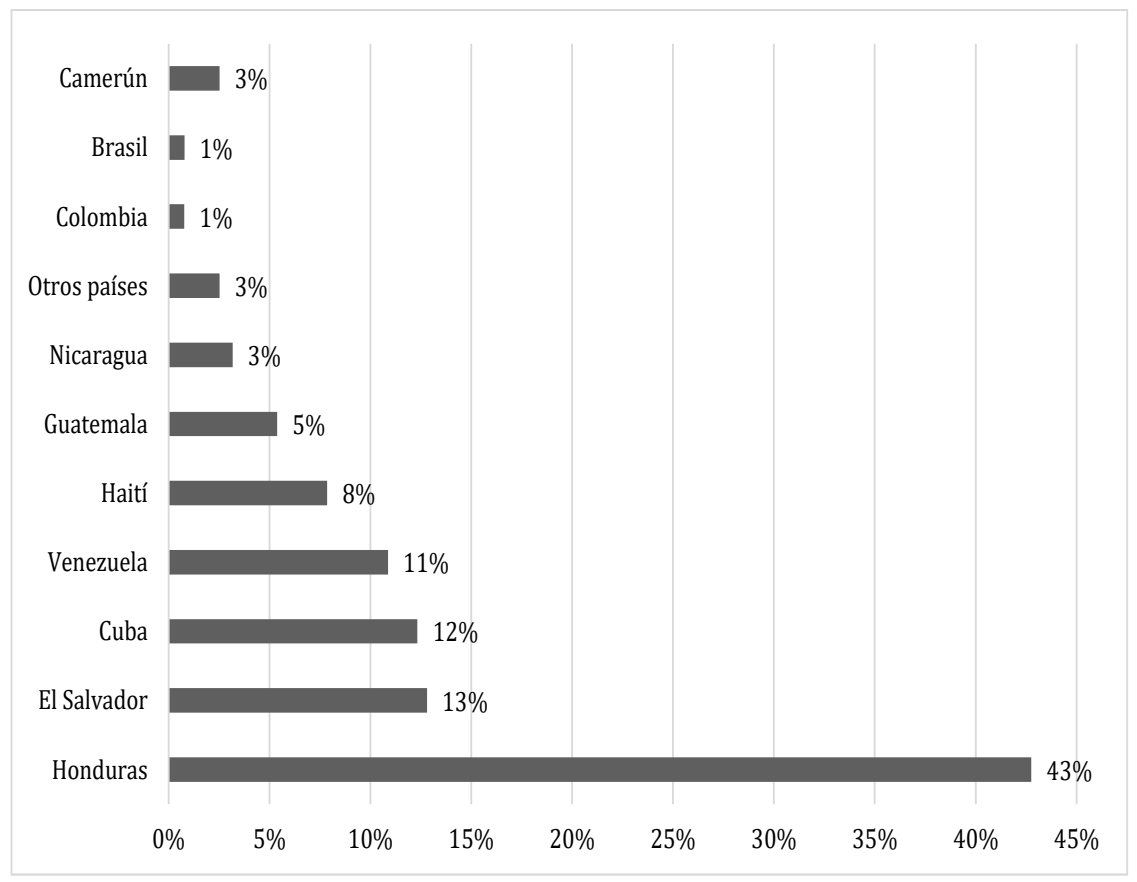

Fuente: Elaboración propia con datos de Comisión Mexicana de ayuda a refugiados, 2019.

Las tarjetas de residentes temporales y permanentes son documentos que permiten la estancia de extranjeros en México, la primera aplica a personas extranjeras que pretenden internarse a México en la condición de residente temporal con el objeto de permanecer por un tiempo mayor a 180 días y menor a cuatro años, si es mayor a ese tiempo tendrá que tramitar una visa de residencia permanente (Gobierno de México, 2020). Estos documentos pueden solicitarse en cualquier representación consular de México y le permite al extranjero trabajar en el país. El tramité debe hacerse dentro de los siguientes 30 días naturales contados a partir de su ingreso al territorio nacional, esta 
tarjeta de residencia certifica su estancia legal y le permite permanecer en territorio nacional(Gobierno de México, 2020). En la Figura 3 se puede apreciar que los migrantes de Venezuela son quienes tienen el mayor porcentaje con renovación de tarjetas de residencia temporal (5.3 por ciento) y de residencia permanente ( 5.8 por ciento), lo anterior responde a la situación económica, política y social que vive ese país. Estados Unidos presenta el mayor porcentaje de tarjetas de residencia temporales con 4.5 por ciento, por su parte países de Centroamérica como El Salvador, Honduras y Guatemala presentan muy bajos porcentajes en las tarjetas de residencia temporal y más significativa en aquellas residencias permanentes. Lo anterior permite identificar una residencia más prolongada de migrantes centroamericanos a diferencia de otras poblaciones extranjeras residentes en México.

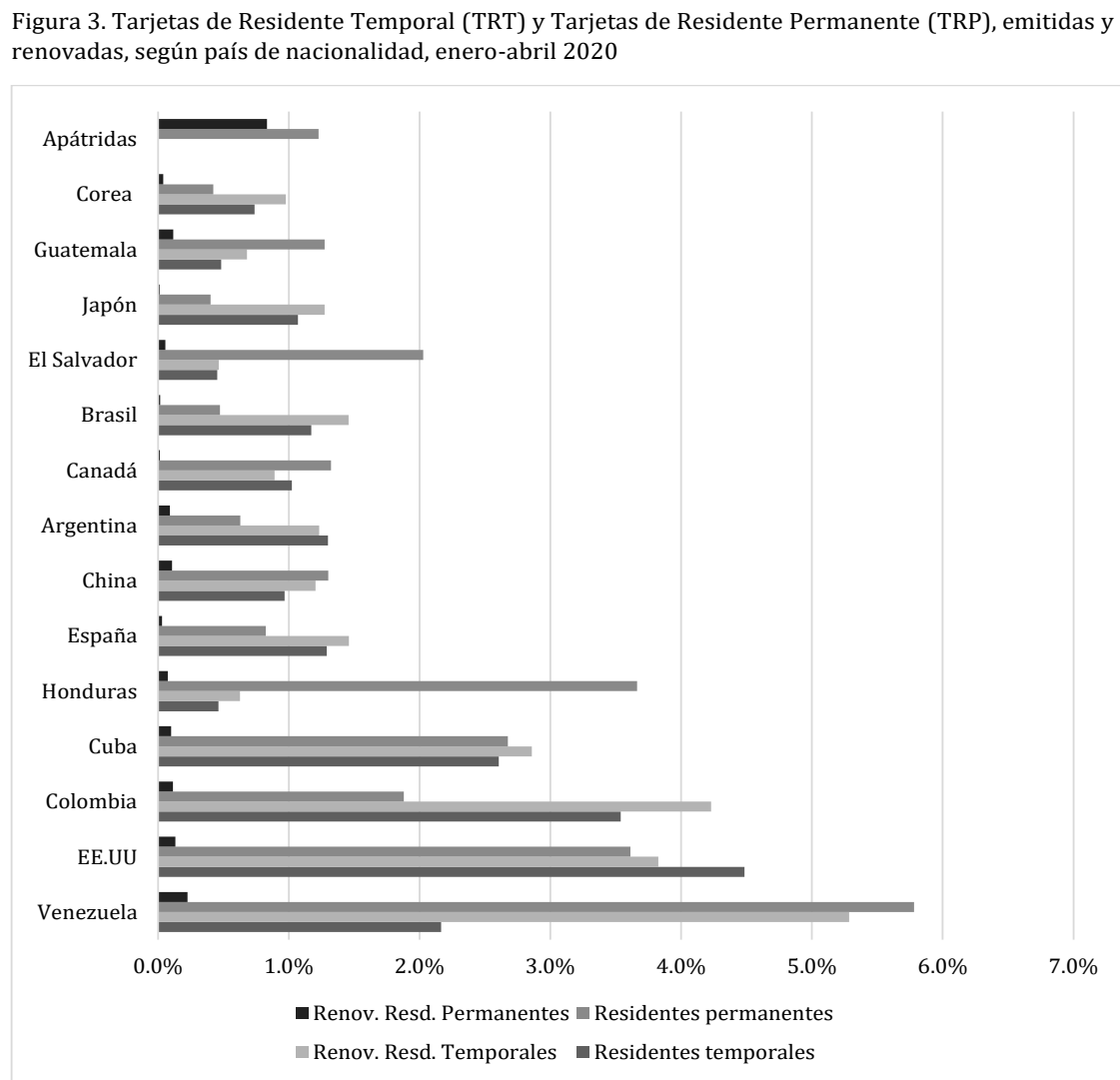

Fuente: Elaboración propia con datos de la Unidad de Política Migratoria, Registro e Identidad de Personas, SEGOB, con base en el Boletín Mensual de Estadísticas Migratorias 2020. 
México ha adquirido relevancia como país de tránsito en la movilidad de personas al vecino país de Estados Unidos; ya sea cual fuere el estatus legal migratorio. De acuerdo con lo que estipula el Instituto Nacional de Migración el tránsito de migrantes centroamericanos se ha intensificado en las últimas dos décadas de manera sustancial. El migrante centroamericano representa un grupo cuya finalidad es llegar a Estados Unidos, por lo que se establecen por tiempo indefinido o simplemente van de paso por México. Aunado a ello, estos migrantes, sus familias y entornos sociales dadas las circunstancias políticas, sociales e institucionales, son orillados a convertir los lugares de residencia provisional, en los de residencia definitiva (Fuentes \& Ortiz, 2012).

Llegue de Honduras en abril de 2019 junto a mis tres hijos, huíamos de la violencia y de la inseguridad pues las bandas de donde vivíamos en San Pedro nos obligaron a huir, ya llevaban meses mis hijos sin ir a la escuela tuvimos que salir sin nada pues los pandilleros nos dieron solo 24 horas para salir, al principio queríamos ir a Estados Unidos, pero está difícil, ahora estoy en Tula y ya tengo mi residencia temporal, las cosas deben ir ahora mejor (Entrevista a María Cruz, con la autora, 22 de enero de 2020).

La violencia y falta de oportunidades son elementos detonadores e importantes para la migración, estos migrantes se sintieron inseguros en su país y con escasas oportunidades económicas. Quienes han podido salir lo han hecho cruzando fronteras de forma ilegal, sin planes de regresar debido a la violencia e inseguridad como la razón principal. Por ello, en la migración la violencia es un factor decisivo para salir de sus lugares de origen y en este caso establecerse en México, ya sea mediante solicitud de asilo o refugio, buscando obtener su residencia temporal o permanente.

Yo he pedido refugio en México, pero es muy tardado y con todos los trámites y no he seguido, por eso pue mi situación, fui a consulado me dieron papeles, me enviaron a migración, me mandaron a COMAR de ahí ya no volví (Entrevista a Juana Ortiz con la autora, 3 de febrero de 2020).

\section{VOLUMEN Y CARACTERÍSTICAS DE LA POBLACIÓN Centroamericana en MÉXICo}

Según datos proporcionados por el INEGI (2015) se reportan 1'007,063 personas nacidas en el extranjero con residencia en México. Sin embargo, de esta cifra pudiera tener un subregistro debido a la 
gran cantidad de inmigrantes indocumentados que transitan por sólo unos días en México y cuyo destino final es Estados Unidos.

Tabla 1. Extranjeros residentes en México 2015

\begin{tabular}{lrr}
\hline Países & Población & Porcentaje \\
\hline Estados Unidos & 739,168 & 73.3 \\
Guatemala & 42,874 & 4.26 \\
España & 22,646 & 2.25 \\
Colombia & 18,735 & 1.86 \\
Venezuela & 15,664 & 1.56 \\
Argentina & 14,747 & 1.46 \\
Honduras & 14,544 & 1.44 \\
Cuba & 12,768 & 1.27 \\
El Salvador & 10,594 & 1.05 \\
Canadá & 9,816 & 0.97 \\
Otros países & 105,507 & 10.48 \\
Total & $1^{\prime} 007,063$ & 100.00 \\
\hline
\end{tabular}

Fuente: elaboración propia con datos de la Encuesta Intercensal (2015).

De acuerdo con las cifras proporcionadas por el INEGI, se puede observar que dentro del grupo de extranjeros que residía en México en el año 2015 predominaban los estadounidenses con 73.4 por ciento (esto es a consecuencia de la tradición histórica existente de la migración México - Estados Unidos, donde persiste un vínculo entre la población de ambos países), por su parte con gran presencia se ubican a los guatemaltecos 4.3 por ciento, españoles 2.2 por ciento, colombianos 1.9 por ciento y venezolanos 1.6 por ciento principalmente. De ahí se desprende que alrededor del 8 por ciento de la población de extranjeros residentes en México corresponde a centroamericanos, mismos que han adquirido gran importancia en los últimos años (ver Figura 4), internándose en la frontera sur de México con el propósito de llegar a los Estados Unidos. Por lo cual hoy en día constituye uno de los retos del gobierno mexicano en materia de derechos humanos. 
Figura 4. Tasa de crecimiento de la población centroamericana en México, 1990-2015

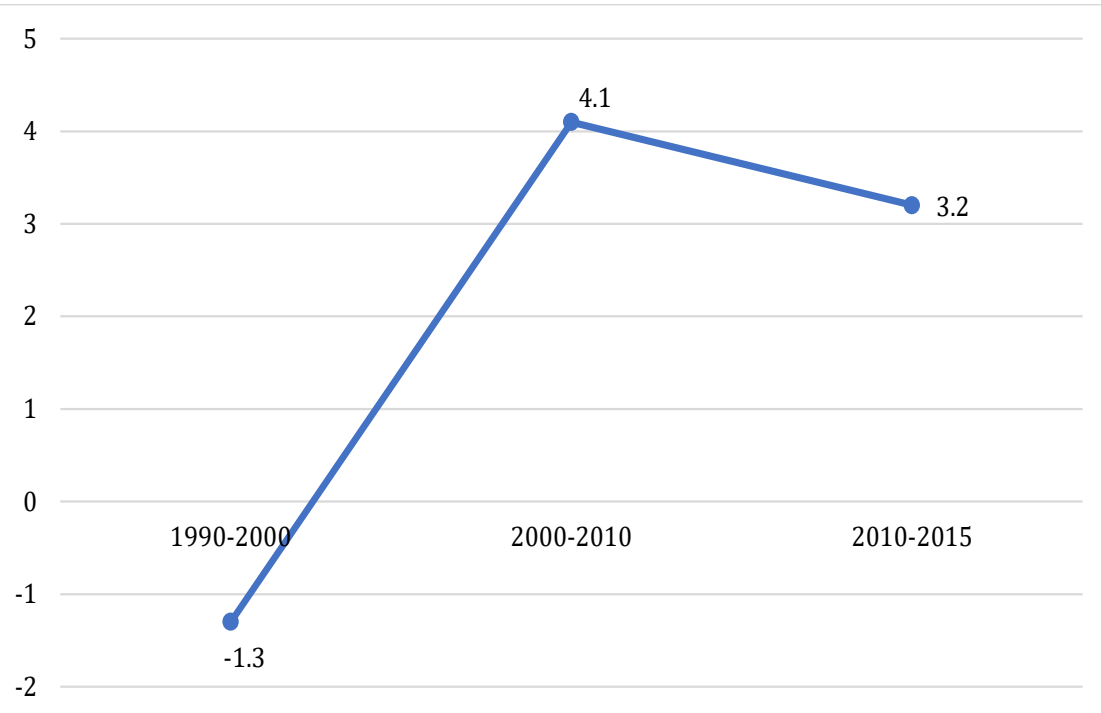

Fuente: Elaboración propia con datos del Censo de Población y Vivienda (1990), Censo de Población y Vivienda (2000), Censo de Población y Vivienda (INEGI, 2010) y la Encuesta Intercensal (2015).

La población centroamericana fue de alrededor de 79,030 residentes los cuales de acuerdo con el país de origen se observa como ya se ha señalado una predominancia de guatemaltecos con 54.3 por ciento, es decir, poco más de la mitad de esta población, seguido de hondureños con 18.4 por ciento y salvadoreños con 13.4 por ciento principalmente (ver Tabla 2).

A continuación, se hace un análisis del perfil sociodemográfico del migrante centroamericano que reside en México y que fue captado en el levantamiento de información de la Encuesta Intercensal del año 2015. Esta población se integra por 46.6 por ciento de hombres y 53.4 por ciento de mujeres, aunque existen diferencias entre países de procedencia. Además, la población residente de Centroamérica es particularmente joven en edades laborales, con una edad mediana de 34 años, coincidiendo con una migración laboral, de donde se observa que menos de diez por ciento están dentro del grupo de edad de 0-14 años y cerca de 84 por ciento está entre los 15 a 64 años, siendo el grupo minoritario el de los adultos mayores con seis por ciento .Por lo anterior, 
se observa que el fenómeno de la migración centroamericana se ha focalizado no solo a adultos sino también a niños y jóvenes migrantes.

Tabla 2. Residentes centroamericanos en México según lugar de nacimiento, 2015

\begin{tabular}{lrr}
\hline País & Población & Porcentaje \\
\hline Belice & 2,124 & 2.7 \\
Costa Rica & 3,359 & 4.3 \\
El Salvador & 10,594 & 13.4 \\
Guatemala & 42,874 & 54.3 \\
Honduras & 14,544 & 18.4 \\
Nicaragua & 4,196 & 5.3 \\
Panamá & 1,339 & 1.7 \\
Total & 79,030 & 100.0 \\
\hline
\end{tabular}

Fuente: elaboración propia con datos de la Encuesta Intercensal (2015).

Por nacionalidad se puede ver que los guatemaltecos es el grupo más numeroso que se encuentra con población en edades jóvenes y laborales ya que más de dos terceras partes de su población residente en México y se ubica dentro de las edades productivas (ver Figura 5). Para el año 2015, alrededor de 16.4 por ciento de migrantes centroamericanos se ubica en el grupo de edad de 0 a19 años de edad (si bien se ha incrementado el porcentaje de este grupo de migrantes centroamericanos, en términos generales, es el grupo de edad con menor presencia), en el resto de los años analizados se observa que el mayor porcentaje de migrantes pertenecen al grupo de 20 a 39 años de edad con 47.5 por ciento y pese a lo que podría esperarse, el porcentaje de migrantes de 40 a 64 años con 30.4 por ciento, es superior al de los menores de 19 años. La evidencia empírica señala una migración Centroamericana relativamente joven y en edades productivas y los datos obtenidos de la Encuesta Intercensal permiten identificar esta información. 
Figura 5. Estructura de la población centroamericana residente en México, 2015

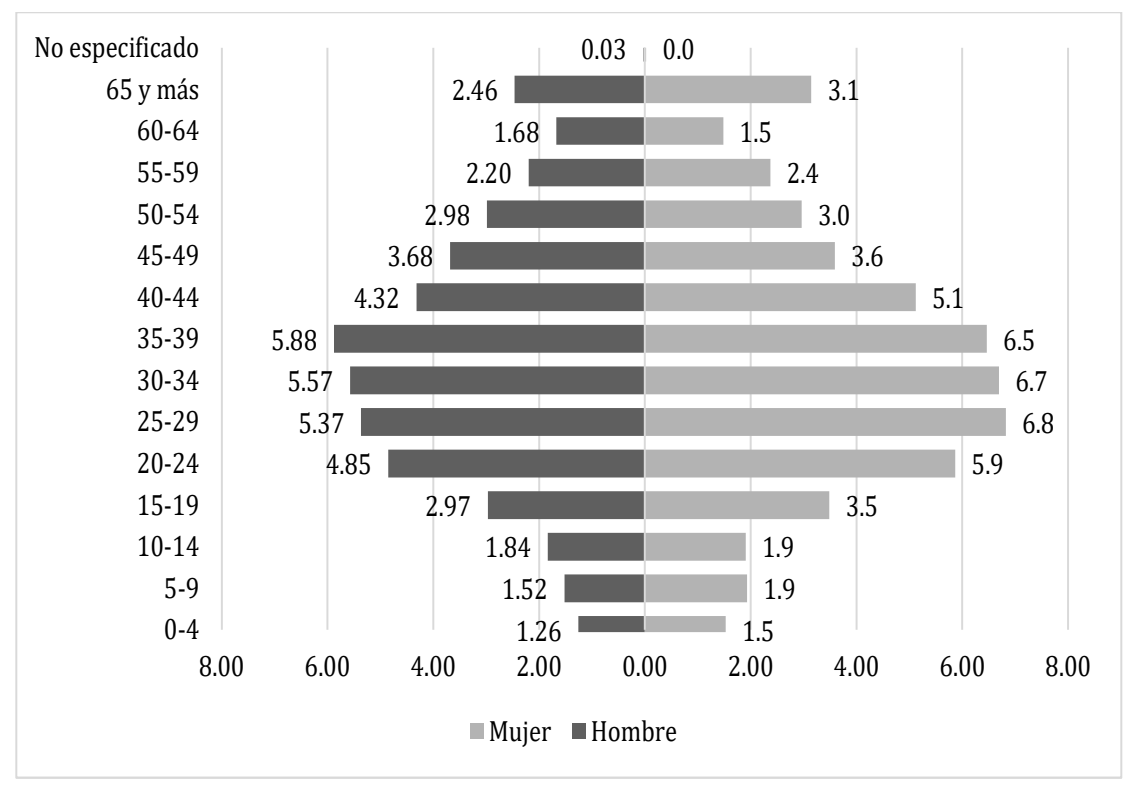

Fuente: Elaboración propia con datos de la Encuesta Intercensal (2015).

La ubicación geográfica de los migrantes centroamericanos dentro del país es de la siguiente manera: principalmente en Chiapas con 43 por ciento lo cual es esperado dada la vecindad geográfica con México, Quintana Roo con 9.3 por ciento, ambas cifras en conjunto suman poco más de la mitad de esta población centroamericana residente en México, en un segundo grupo se encuentran las entidades de Ciudad de México con 6.9 por ciento, Baja California con 4.8 por ciento, Campeche con 4.6 por ciento, Estado de México con cuatro por ciento, Nuevo León con 3.5 por ciento y Jalisco con 2.5 por ciento, en su conjunto suman el 26.3 por ciento, el restante 21.4 por ciento se distribuye en el resto de las entidades del país (INEGI, 2015) (Ver mapa 1). La vivienda, el mercado laboral, el acceso a la información, salud, transporte y educación son factores sobre los cuales los migrantes tienen poco dominio, pero que son decisivos para ubicarse dentro del territorio nacional. 


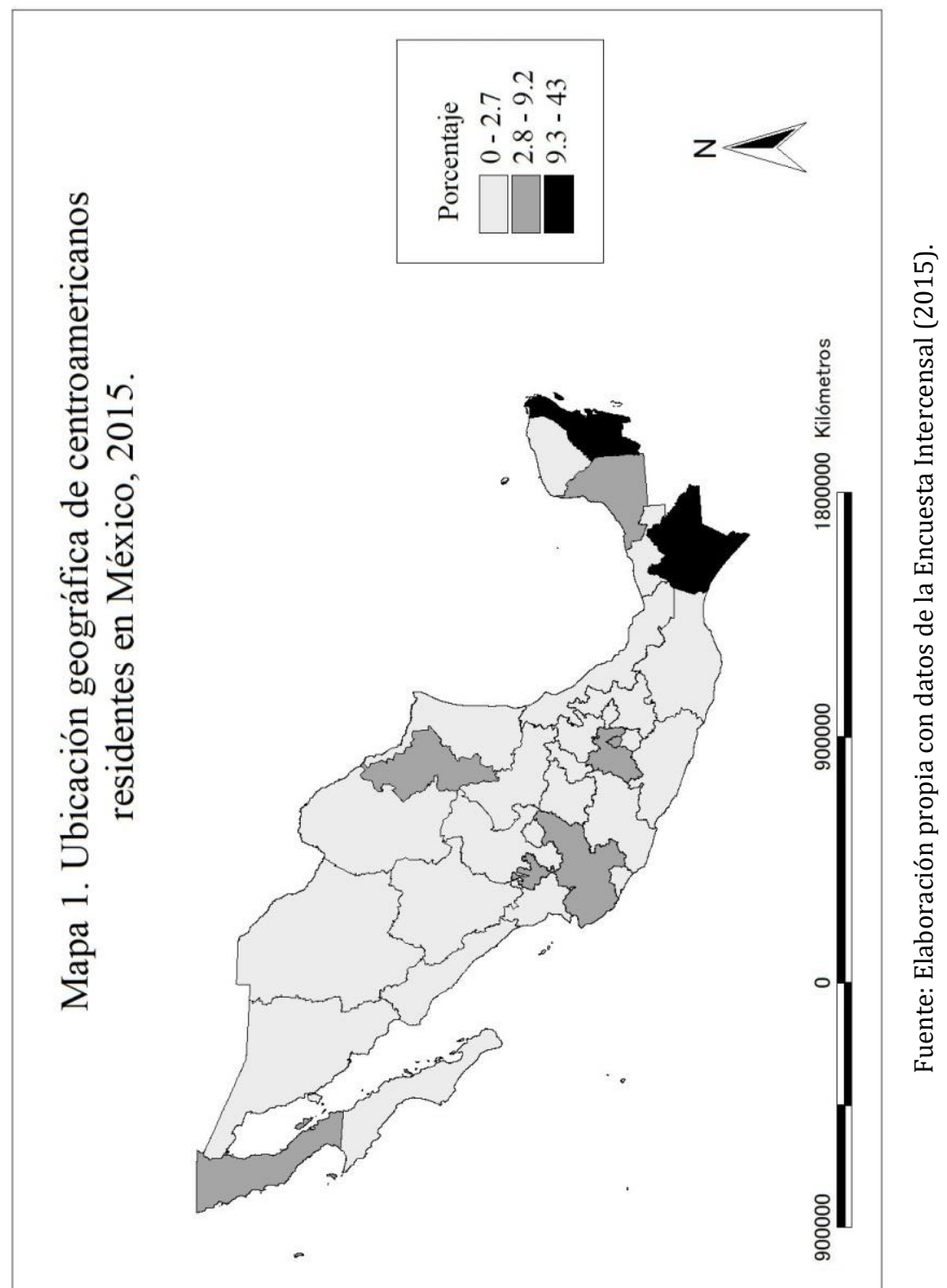


La población centroamericana en cuanto a su situación conyugal se observa que predominan los de unión libre con 41.8 por ciento, seguido de los casados con 27.7 por ciento, solteros con 21.3 por ciento y separados con 4.5 por ciento (Ver Figura 6). Esta situación permite identificar una migración mayoritariamente de personas casadas o en unión libre con 69.5 por ciento en comparación con los solteros, es decir, solo uno de cada cinco migrantes se encuentra en esa situación, por lo que se puede apreciar que estos flujos están constituidos por personas que, si bien, o son jefes de hogar o son esposos o esposas dentro de la estructura familiar. Por otro lado, es posible que el restante porcentaje se explique en parte por una menor dependencia económica del hogar respecto a los ingresos obtenidos por el grupo antes señalado.

Figura 6. Situación conyugal de la población centroamericana residente en México, 2015

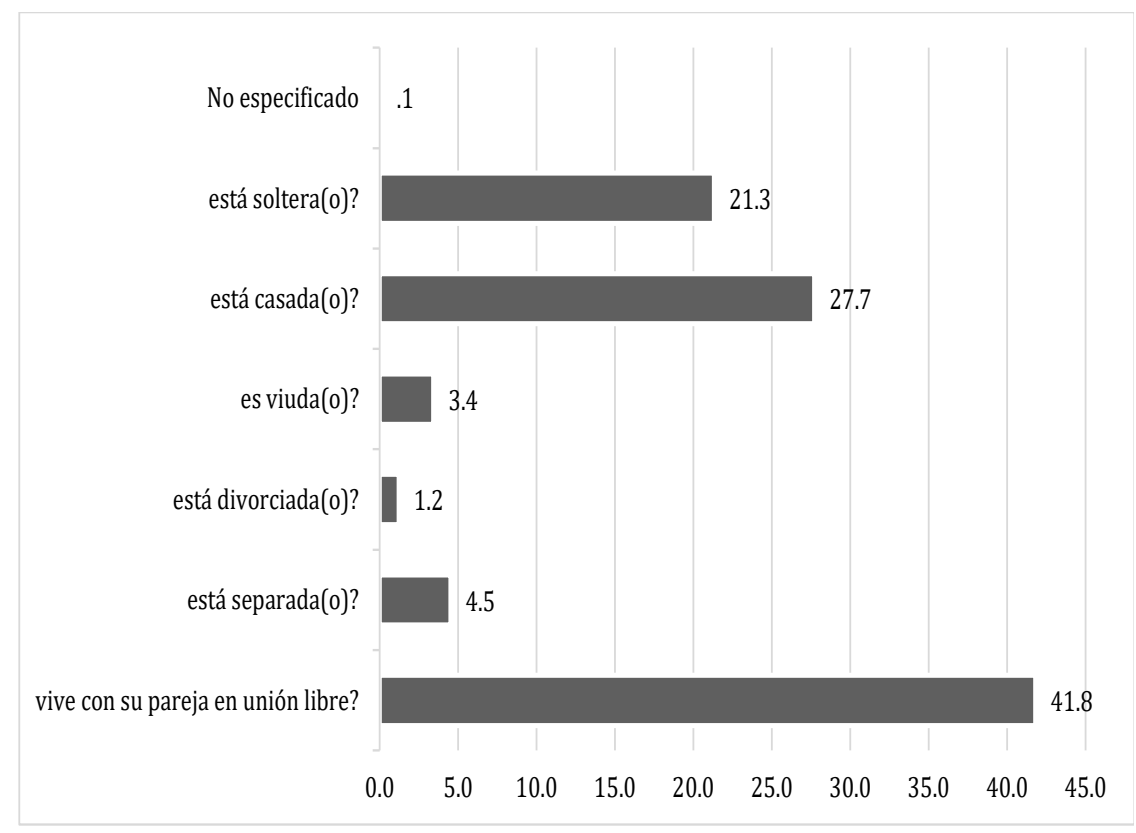

Fuente: Elaboración propia con datos de la Encuesta Intercensal (2015).

Se observa un nivel educativo bajo con una escolaridad mediana de seis años, además alrededor de 40.6 por ciento solo contaba con primaria o menos, seguido con 18.3 por ciento de quienes no tenían instrucción alguna; es decir, alrededor de tres de cada cinco migrantes se ubicaba en esta situación, y solo 12.4 por ciento tenía licenciatura 
o más grados escolares, lo anterior corrobora que esta población migrante es poco calificada y con bajos niveles educativos, consecuencia de condiciones económicas o de seguridad de los lugares origen (Ver Figura 7).

Figura 7. Escolaridad de la población centroaméricana residente en México, 2015

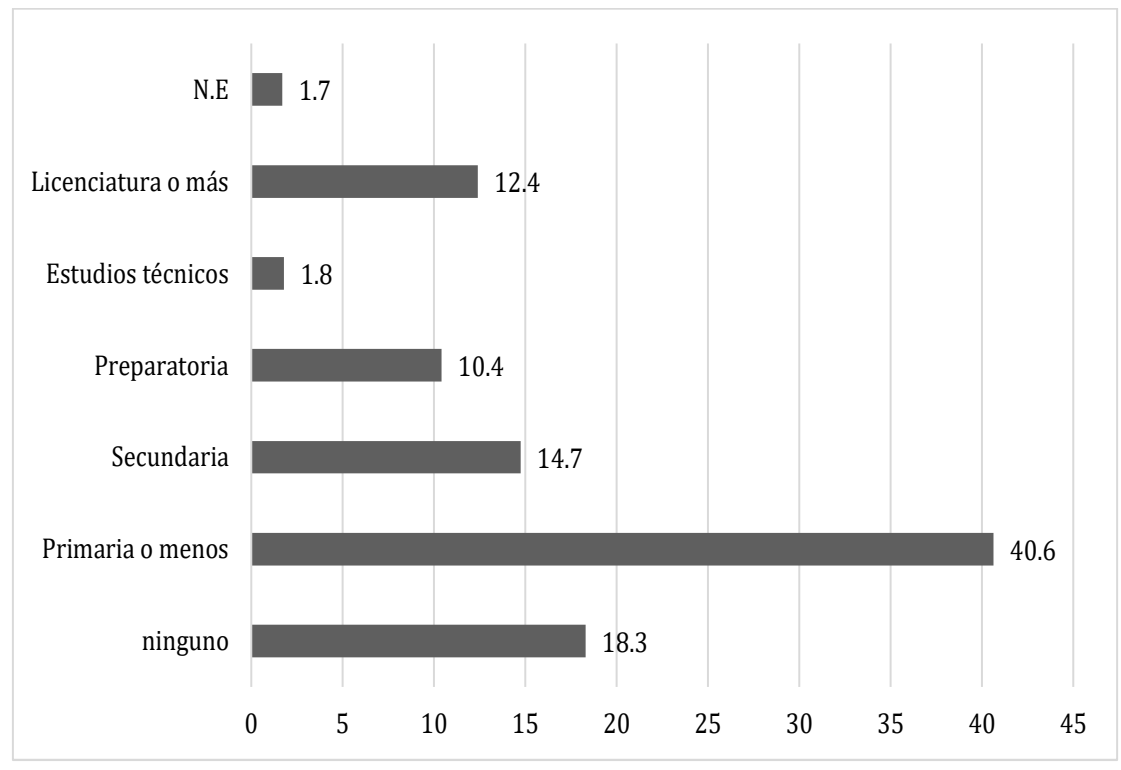

Fuente: Elaboración propia con datos de la Encuesta Intercensal (2015).

En términos generales, para la información estadística analizada la distribución por niveles de escolaridad indica comportamientos donde concentra en su mayoría niveles bajos de educación, con solo 12.4 por ciento en los niveles con Licenciatura o más. Lo anterior permite dar cuenta sobre la selectividad de los migrantes, donde se presentaron los datos del nivel educativo muy bajo.

Mi esposo decía que no quería llegar a Estados Unidos lo que quería era salir de Honduras a donde sea, salimos huyendo de las pandillas, nuestro hijo ni a la escuela lo pudimos ya mandar, allá es muy difícil estudiar, yo ni la primaria pude terminar, no te dejan siempre te pueden acosar, y si les gustas como sus mujeres te pueden llevar (Entrevista a Ana Dimas, con la autora, 16 de enero de 2020).

Salimos por darles una mejor oportunidad a mis hijos por darles una mejor vida un lugar donde vivir, sin estar en un cartel, una mara o una pandi- 
lla, ese es mi sueño darles estudios a mis hijos, allá no se puede casi nadie estudia, no importa me sacrifiqué yo,para que ellos tengan una mejor vida, por eso salimos huyendo de Honduras a como diera lugar (Entrevista a Mario Vázquez, con la autora, 22 de enero de 2020).

Una población altamente productiva y con elevados niveles de escolaridad tendrá mejores oportunidades de colocarse laboralmente en el lugar destino. Sin embargo, no es el caso de los residentes centroamericanos, ya que ellos perciben en promedio alrededor de 6,285.7 pesos.

El 50.4 por ciento de la población se encontraba laborando al momento de la encuesta, seguido de 27.9 por ciento que se consideraban trabajadores del hogar, 4.2 por ciento eran estudiantes y el restante se ubicaba buscando trabajo, eran jubilados o estaban de licencia. Con respecto a su condición laboral y acceso al trabajo, se identifica que alrededor de 84.3 por ciento de centroamericanos es población económicamente activa, esto se explica por la alta migración existente de paso a Estados Unidos en busca de mejores oportunidades laborales (INEGI, 2015).

Yo acá he vivido en Tula llevo ya casi dos años, mi esposa llego con mis niños el 22 de noviembre del 2019, y pue le gusta esta Ciudad porque hay más seguridad es grande y los niños ya los mandamos a la escuela, ya están en el kínder y en primaria, yo todas las mañanas me levanto a las 6 de la mañana y me voy caminando aquí cerca el albergue la casa del migrante, para irme a trabajar, quien me ocupe en algún lugar, es lo que hago todos los días (Entrevista a Juan Hernández, con la autora, 25 de febrero de 2020 ).

En relación con la ocupación principal de los centroamericanos residentes en México es variada, alrededor de 50 por ciento se desempeña como empleado u obrero, trabajador por cuenta propia 26 por ciento, esta diversificación de las ocupaciones se relaciona con la mayor oferta de empleos en el ámbito urbano en donde el sector terciario predomina reflejado en las actividades desempeñadas. Asimismo, 11 por ciento se desempeña como jornalero o peón donde sus actividades son propias del mercado laboral agrícola (INEGI, 2015), y en éste generalmente predomina la precariedad, la inestabilidad, la falta de prestaciones y condiciones de trabajo y explotación a que pueden ser sometidos estos migrantes. 
Referente a los servicios de salud se vislumbra una alta vulnerabilidad debido a que poco más de la mitad de esta población no se encontraba afiliada a algún servicio de salud, 21.5 por ciento al seguro popular y solo 13.3 y 1.7 por ciento al Instituto Mexicano del Seguro Social (IMSS) y al Instituto de Seguridad Social al Servicio de los Trabajadores del Estado (ISSSTE), respectivamente. Lo que permite identificar que no contaban con algún empleo que les brindara estos servicios.

La población migrante centroamericana trabajadora se caracteriza por ubicarse en empleos precarios, de baja calificación e informales, solo algunos que trabajan dentro del sector formal poseen prestaciones laborales tales como: aguinaldo solo 13.5 por ciento lo tiene, vacaciones diez por ciento, licencia por incapacidad 8.6 por ciento, reparto de utilidades cinco por ciento, sistema de ahorro para el retiro (SAR O AFORE) 7.1 por ciento y acceso al crédito para la vivienda 6.8 por ciento (INEGI, 2015).

No tengo prestaciones, ni aguinaldo ni nada de eso, tengo trabajo me voy diario a trabajar en lo que se puede, lavando carros allá y acá, estoy ahí con un señor vamos pa delante que vamos saliendo poco a poco y me voy ubicando bien en lo que sea para mantener a mi familia (Entrevista a Mario Vázquez, con la autora, 22 de enero de 2020).

Desde que llegue estoy trabajando en la panadería, ayudando a hacer el pan de dulce y los bolillos, pero como no tengo papeles no tengo ayudas ni prestaciones de nada, pero eso sí, tengo mi sueldo que es poco, pero es mejor que nada (Entrevista a Teresa Bautista, con la autora, de abril de 2020)

De prestaciones... no pue nada ... yo lavo y plancho ropa, solo eso por la falta de papeles, y pue tengo que mantener a mis hijos de 8 y 5 años, es lo único que no me gusta aquí no hay trabajo, a los migrantes no nos dan trabajo me gustaría arreglar papeles pue a mis hijos los tengo aquí sin estudiar (Entrevista a Juana Ortiz con la autora, 3 de Febrero de 2020).

La relación de la ocupación del migrante y los servicios de salud van estrechamente relacionados, ya que, si tienen un trabajo precario, evidentemente carecerán de cualquier servicio de salud o prestación alguna, siendo esto una situación estructural de vulnerabilidad.

\section{CONCLUSIONES}

Los procesos migratorios provenientes del Sur han adquirido gran importancia y especialmente el de origen de Centroamérica, esta migración se ha caracterizado por ser un proceso de movilidad espacial y 
social, cuyo objetivo primordial es mejorar las condiciones de vida con respecto a su lugar de origen. La migración de Centroamericanos y por consecuencia la residencia de estos mismos en México es un hecho que continuará incrementándose y se prolongará por mucho tiempo, así como sus consecuencias.

La movilidad de centroamericanos a su paso por México es un tema ubicado dentro de los temas prioritarios de los gobiernos y la sociedad en general convirtiéndose en eje del escenario político, social y económico a nivel global. De tal manera, que ha adquirido importancia como país de tránsito y residencia en la movilidad de personas ya sean documentadas o indocumentadas, e inclusive hoy en día como país receptor de estos migrantes cuyo camino se vio truncado a su meta final Estados Unidos. En ese sentido esta investigación se centró en el estudio de la población residente centroamericana en México.

Diariamente miles de centroamericanos cruzan la frontera con la finalidad de llegar a los Estados Unidos, siendo el lugar estratégico para entrar al país la frontera Sur, constituida por Chiapas, Quintana Roo, Tabasco, y Campeche, las dos primeras concentran el mayor número de centroamericanos residentes. Sin embargo, un alto porcentaje de ellos no siempre logra el objetivo, optando por establecerse en México (por tiempo indefinido o simplemente de paso), modificando el entorno de su lugar de destino, ya que se enfrentan a nuevas costumbres, formas de vida, cultura, relaciones sociales, situaciones económicas, políticas, etcétera.

El análisis sociodemográfico permite conocer el perfil de este migrante que ha llegado a México. Se señala que es una población joven en edades productivas y con bajos niveles de escolaridad, y puestos de trabajo de empleados o jornaleros principalmente.

\section{REFERENCIAS BIBLIOGRÁFICAS}

Alvarez, S. (2011). Migración indocumentada en tránsito: la cara oculta de los procesos migratorios contemporáneos, Consejo Latinoamericano de Ciencias Sociales-CLACSO, pp. 5-18. Buenos Aires.

Bautista, A., \& Ibarra, V. (2014). "La movilidad cotidiana: espejo de la forma urbana”, en Coyuntura Demográfica, 71-82.

Carrasco, González, G. (2013). "La migración centroamericana en su tránsito por México hacia los Estados Unidos", en Revista Alegatos. No. 82, abril/mayo. pp. 169-193. 
Consejo Nacional de Población (2016). Prontuario sobre movilidad y migración internacional. Dimensiones del fenómeno en México. Ciudad de México: Consejo Nacional de Población/Secretaría de Gobernación.

Colegio de la Frontera Norte (2019). Caravana de migrantes centroamericanos en Reynosa y Matamoros, 2019. Reynosa, Tamaulipas, México: COLEF.

COMAR Comisión Mexicana de ayuda a refugiados. (agosto de 2019). COMAR. Obtenido de https://www.gob.mx/cms/uploads/attachment/ file/493180/REPORTE_CIERRE-DE-AGOST0-2019.pdf

Consejo Nacional de Población/Secretaría de Gobernación. (2016). Prontuario sobre movilidad y migración internacional. Dimensiones del fenómeno en México. Ciudad de México: Consejo Nacional de Población/Secretaría de Gobernación.

Expansión (2019). Violencia, pobreza y cambio climático, causas de la migración en Centroamérica. Recuperado el 10 de Octubre de 2020 de https://politica.expansion.mx/mexico/2019/06/18/violencia-pobreza-y-cambio-climatico-causas-de-la-migracion-en-centroamerica.

Fuentes, G., \& Ortiz, L (2012). "El migrante centroamericano de paso por México, una revisión a su condición social desde la perspectiva de los derechos humanos", en Revista Convergencia, vol.19, núm. 58, Toluca ene./ abr, pp.157-182.

Gobierno de México (24 de junio de 2020). https://www.gob.mx/sre/acciones-y-programas/visa-de-residencia-temporal. Obtenido de https:// www.gob.mx/sre/acciones-y-programas/visa-de-residencia-temporal: https://www.gob.mx/sre/acciones-y-programas/visa-de-residencia-temporal

Hernández, H. O. (2019). Caravana de migrantes centroamericanos en Reynosa y Matamoros, 2019. Tamaulipas, México: Colegio de la Frontera Norte.

INEGI (1990). Censo de Población y Vivienda 1990. México: Instituto Nacional de Estadística y Geografía.

INEGI (2000). Censo de Población y Vivienda. México: Instituto Nacional de Estadística y Geografía.

INEGI (2010). Censo de Población y Vivienda 2010. Ciudad de México: Instituto Nacional de Estadística y Geografía.

INEGI (2015). Encuesta Intercensal 2015. Aguascalientes: Instituto Nacional de Estadística y Geografía.

Instituto Nacional de Migración (18 de 07 de 2019). https://www.inm. gob.mx. Obtenido dehttps://www.inm.gob.mx/gobmx/word/index.php/ tema-migratorio-180719/ 
López, N. (2018). Procesos migratorios en la Centroamérica del siglo XXI. México: Universidad Nacional Autónoma de México.

Migration Policy Institute (MPI) (15 de Agosto de 2019). Migration Policy Institute (MPI). Obtenido de https://www.migrationpolicy.org/article/inmigrantes-centroamericanos-en-los-estados-unidos

Naciones Unidas (2016). Situación de los migrantes en tránsito. Ginebra, Suiza: Naciones Unidas.

Naciones Unidas (28 de junio de 2020). Naciones Unidas derechos humanos. Obtenido de Naciones Unidas derechos humanos: https://www. ohchr.org/Documents/Issues/Discrimination/LGBT/FactSheets/UNFEFactSheet_RefugeAsylum_ES.pdf

Nájera, J. (2015). "El complejo estudio de la actual migración en tránsito por México: actores, temáticas y circunstancias", en Migraciones Internacionales.

Organización Internacional para las migraciones OIM (1 de Octubre de 2018). Oficina Regional para Centroamérica, Norteamérica y el Caribe. Obtenido de https://rosanjose.iom.int/site/es/caravanas-migrantes

Ramírez, B. (2015). "Alcances y dimensiones de la movilidad, aclarando conceptos", en B. Ramírez, Debates y estudios de la movilidad laboral en la región centro del país: alcancez y dimensiones desde México (págs. 25-38). Ciudad de México: Universidad Autonóma Metropolitana.

Romero, G., \& Lugo, D. (2018). "El estado del arte de la movilidad del transporte en la vida urbana en las ciudades latinoaméricanas", en Transporte y territorio, 133-157.

Entrevistas

Bautista, Teresa; 28 años, hondureña con un año de residir en México, entrevista realizada el 1 de abril de 2020 en Tuxtla, Gutiérrez Chiapas, México.

Cruz, María; 34 años, hondureña con4 años de residir en México, entrevista realizada el 22 de enero de 2020 en Tula Hidalgo, México.

Dimas, Ana; 21 años, hondureña con año y medio residiendo en México, entrevista realizada el 16 de enero de 2020 en Tuxtla Gutiérrez, Chiapas, México.

Hernández, Juan; 33 años guatemalteco con 3 años y medio residiendo en México, entrevista realizada el 25 de febrero de 2020 en Tula, Hidalgo, México.

López, José; 32 años, hondureño con 2 años de residir en México, entrevista realizada, el 6 de marzo de 2020 en Tuxtla, Gutiérrez Chiapas, México.

Ortiz, Juana; 30 años, salvadoreña con 2 años residiendo en México, entrevista realizada el3 de febrero de 2020, en Tula Hidalgo, México. 
Vázquez, Mario; 35 años, hondureño con 2 años residiendo en México, entrevista realizada el 22 de enero de 2020en Tula Hidalgo, México.

\section{RESUMNEN CURRICULAR DE LA AUTORA}

\section{Laura Myriam Franco Sánchez}

Doctora en urbanismo, maestra en estudios regionales y licenciada en Economía; adscrita como profesora investigadora en la Universidad Autónoma del Estado de Hidalgo en el área académica de Sociología y Demográfía. Dentro de sus publicaciones se ubican: Migración y movilidad laboral entre las zonas metropolitanas de la región centro de México; Perfil sociodemográfico de los commuters en las zonas metropolitanas del estado de Hidalgo; Evaluación del crecimiento de la población y transformación del uso del suelo urbano en la Zona Metropolitana de Pachuca, México; Un nuevo paradigma de la migración venezolana a México, entre otros. Temas de interés: movilidad, migración y desarrollo urbano.Distinguida con el nombramiento del Sistema Nacional de Investigadores Nivel I.

Dirección electrónica: Ifranco@uaeh.edu.mx

Registro ORCID: https://orcid.org/000-0002-0676-9510 